\title{
Preliminary evaluation and discussion of the safety of left innominate vein resection
}

\author{
Hai-Qiang Wang ${ }^{1 \#}$, Feng Tian ${ }^{1 \#}$, Miao Wei ${ }^{2 \#}$, Lian-Hong Chen ${ }^{1}$, Shao-Yi Cheng ${ }^{1}$, Zheng Feng ${ }^{1}$ Jun Ma $^{1}$, \\ Tian-Yi Zhang ${ }^{1}$, Yi-Ze Guo ${ }^{1}$, Xun-Liang Yin $^{1}$, Zheng-Wei Zhao ${ }^{1}$, Yong-An Zhou ${ }^{1}$ \\ ${ }^{1}$ Department of Thoracic Surgery, Tangdu Hospital, Air Force Medical University (Fourth Military Medical University), Xi'an 710038, China; \\ ${ }^{2}$ Department of Otorhinolaryngology Head and Neck Surgery, Xi'an Children's Hospital, Xi'an 710000, China \\ Contributions: (I) Conception and design: HQ Wang, F Tian, M Wei; (II) Administrative support: ZW Zhao, YA Zhou; (III) Provision of study \\ materials or patients: HQ Wang, LH Chen, SY Cheng, Z Feng, J Ma, TY Zhang, YZ Guo, XL Yin; (IV) Collection and assembly of data: HQ Wang, \\ LH Chen, SY Cheng, Z Feng, J Ma, TY Zhang, YZ Guo, XL Yin; (V) Data analysis and interpretation: HQ Wang, F Tian, M Wei; (VI) Manuscript \\ writing: All authors; (VII) Final approval of manuscript: All authors. \\ \#These authors contributed equally to this work. \\ Correspondence to: Zheng-Wei Zhao; Yong-An Zhou. Department of Thoracic Surgery, Tangdu Hospital, Air Force Medical University (Fourth \\ Military Medical University), Xi'an 710038, China. Email: zhaowei_zw@qq.com; zhou.yongan@163.com.
}

Background: To evaluate the safety of resection of anterior mediastinal lesions involving the left
innominate vein (LIV) and analyze the risk factors affecting LIV resection safety.
Methods: Patients who underwent anterior mediastinal lesion and LIV resection from January 2010 to
December 2018 in the Department of Thoracic Surgery of Tangdu Hospital, Air Force Medical University,
were followed up, and preoperative, intraoperative and postoperative factors were analyzed.
Results: Forty-eight patients who underwent anterior mediastinal lesion and LIV resection from January
2010 to December 2018 , except for 2 who died of lung infection-induced respiratory failure, were followed
up, with an average follow-up time of 32 months (range, 6-72 months). Postoperative: in 31 cases (67.39\%),
patients did not manifest LIV resection-associated complications; in 15 cases (32.61\%), patients manifested
mild LIV resection-associated complications; no patient manifested severe LIV resection-associated
complications. The average operation time, average blood loss and average hospitalization time were
155.17 min, 324.13 mL and 11.83 days, respectively. Univariate analysis showed that the degree of LIV
invasion and surgical approach were risk factors for predicting LIV resection safety.

Conclusions: For anterior mediastinal lesions involving the LIV, LIV resection is a simple, safe and effective surgical procedure.

Keywords: Anterior mediastinal lesion; resection of left innominate vein (resection of LIV); safety

Submitted Oct 06, 2019. Accepted for publication Dec 17, 2019.

doi: $10.21037 /$ jtd.2020.01.29

View this article at: http://dx.doi.org/10.21037/jtd.2020.01.29

\section{Introduction}

The anterior mediastinum is a narrow area between the anterior edge of the trachea and pericardium and the sternum, including the thymus and large blood vessels that enter and exit the heart. Anterior mediastinal lesions are uncommon but can occur at any age but are more common to those $30-40$ years of age; of anterior mediastinal lesions, thymomas are the most common, followed by mediastinumtype lung cancer, lymphoma, and neuroblastic tumors $(1,2)$. They often involve the large blood vessels of the thoracic cavity, especially the superior vena cava and its branches, and the left brachiocephalic (innominate) vein (LIV), because it crosses the anterior mediastinum, is susceptible to invasion (3-6). For some patients, surgery may be the only feasible approach of achieving long-term survival (7-14). The safety 


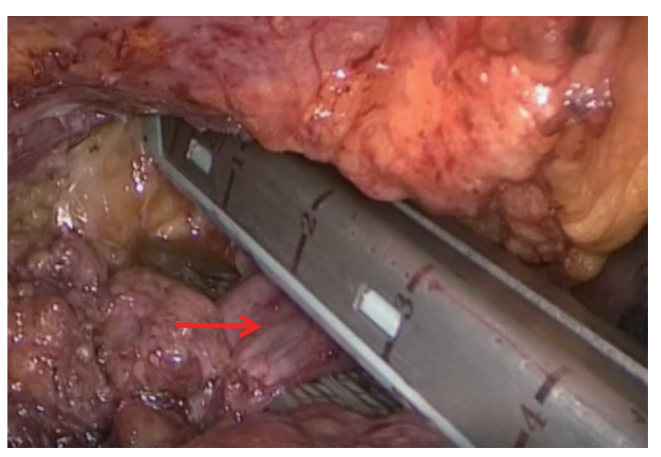

Figure 1 Resection of the invaded distal LIV with a vascular closure device (the arrow shows the LIV). LIV, left innominate vein.

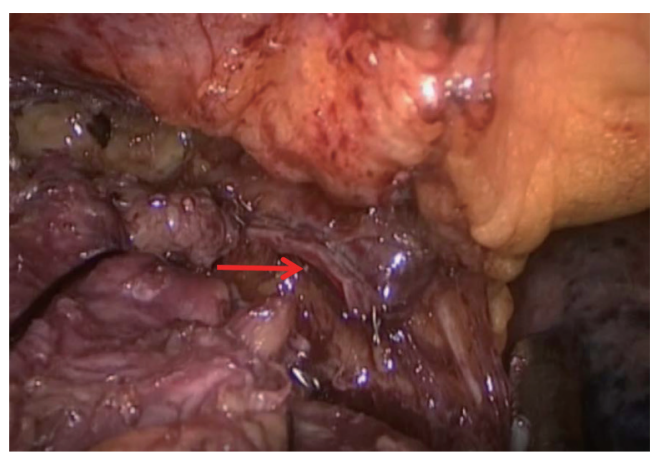

Figure 2 Residual of the LIV (distal end, arrow). LIV, left innominate vein.

and effectiveness of LIV resection in patients with LIV invasion have not been assessed adequately $(6,15,16)$. In this study, we retrospectively analyzed the clinical data of 48 patients with anterior mediastinal lesions involving the LIV who underwent anterior mediastinal lesion and LIV resection, evaluated the safety of LIV resection alone and analyzed the possible influencing factors. The results are reported below.

\section{Methods}

\section{General information}

Data from patients who underwent anterior mediastinal lesion and LIV resection in the Department of Thoracic Surgery of Tangdu Hospital, Air Force Medical University, from January 2010 to December 2018 were retrospectively analyzed. Preoperatively, all patients underwent enhanced chest computed tomography (CT) to determine LIV involvement and stenosis severity. They also underwent complete blood count analysis, blood gas analysis, cardiac ultrasonography, electrocardiography (ECG), and analysis of liver and kidney function, electrolytes, coagulation series, and pulmonary function to exclude surgical contraindications. Our Ethical Committee was informed of the study and did not require approval. All patients gave their informed consent for the study.

\section{Postoperative safety evaluation criteria of LIV resection}

LIV resection may lead to venous return blockage and blood stasis in the drainage area, causing swelling of the left upper limb and face, engorgement of the left jugular vein, varicosity in the left anterior chest wall and upper abdomen, left periorbital edema, conjunctival hyperemia, blurred vision of the left eye, and cerebral edema-derived dizziness, headache, convulsions, and disturbance of consciousness $(15,16)$. "Safety" is defined as no LIV resection-associated complications and no manifestation of the above-described symptoms; "Danger" is defined as the manifestation of severe LIV resection-associated complications (tissue necrosis in the left upper limb, sensory and motor dysfunction, convulsions, impaired vision, and disturbance of consciousness); and "Relative safety" is defined as the manifestation of mild LIV resectionassociated complications (the above-described symptoms but no serious complications).

\section{Categorization of LIV stenosis severity}

With reference to William Sandford's classification of superior vena cava stenosis, we categorized the severity of LIV stenosis as follows: (I) grade i (mild to moderate): partially obstructed LIV (degree of stenosis < $90 \%$ ); (II) class II (severe): almost completely obstructed LIV (degree of stenosis $\geq 90 \%$ ); and (III) grade III (complete stenosis): completely obstructed LIV, i.e., preoperative imaging data show no blood flow in the LIV.

\section{Surgical method and postoperative treatment}

Anterior mediastinal lesions were resected laparoscopically using the "three-hole" operation via the subxiphoid and subcostal arch approach, and the invaded LIV was removed intraoperatively through the proximal and distal end by closure resections with a vascular closure device (Figures 1,2). Thoracotomy (including conversion of laparoscopic 
surgery to open surgery) was used to remove the invaded anterior mediastinal lesion via a vertical incision in the sternum, and the invaded LIV was closed or incised via ligation intraoperatively using a vascular closure device and a suture, respectively, at the proximal and distal ends. All patients were returned to the intensive care unit (ICU) with tracheal intubation and ventilator assistance and injected subcutaneously with low-molecular-weight heparin sodium ( $0.4 \mathrm{~mL} /$ day) within 24 hours after surgery.

\section{Postoperative follow-up and treatment}

Patients were followed up regularly after surgery and subjected to various comprehensive treatments (e.g., radiotherapy and chemotherapy) according to their respective pathological types.

\section{Statistical analysis}

Univariate analysis was performed using the statistical software package SPSS 20.0, in which count data were analyzed using the $\chi^{2}$ and Fisher tests, and the measurement data were analyzed using the $t$-test.

\section{Results (see Tables 1-3)}

\section{General information}

Among the 48 patients, 2 died perioperatively of septic shock derived from pulmonary infection and thus were excluded from the follow-up. The remaining 46 patients were followed up for an average of 32 months (range, 6-72 months); 20 were male, and 26 were female, with an average age of 50.35 years (range, 27-72 years), a preoperative mean body mass index (BMI) of $24.02 \mathrm{~kg} / \mathrm{m}^{2}$ (range, $17-35 \mathrm{~kg} / \mathrm{m}^{2}$ ), a preoperative mean platelet count of $195.29 \times 10^{9} / \mathrm{L}$ [range, $\left.(101-412) \times 10^{9} / \mathrm{L}\right)$ ], and a preoperative mean hemoglobin level of $127.75 \mathrm{~g} / \mathrm{L}$ (range, 109-156 g/L). Nineteen patients manifested hypertension, diabetes, myasthenia gravis, and anemia. Twenty-two patients underwent "three-hole" laparoscopic surgery via the subxiphoid and subcostal arch approach, and 24 patients underwent open surgery via a vertical incision in the sternum, with an average operation time of $155.17 \mathrm{~min}$ (range, 90-260 min), an average blood loss of $324.13 \mathrm{~mL}$ (range, 10-1,000 $\mathrm{mL}$ ) and an average length of hospital stay of 11.83 days (range, 6-19 days). Postoperative pathological examination indicated the following diagnoses: 5 cases of squamous mediastinum, 4 cases of adenocarcinomas mediastinum, 2 cases of seminoma, 2 cases of type A thymoma, 7 cases of type $\mathrm{AB}$ thymoma, 8 cases of type $\mathrm{Bl}$ thymoma, 8 cases of type B2 thymoma, 4 cases of type B3 thymoma, 3 cases of breast cancer, and 3 cases of other diseases. Eleven patients manifested various postoperative complications such as pulmonary infection, pleural effusion, atelectasis, and myasthenic crisis and were all discharged after the complications were treated.

\section{Safety of LIV resection}

Postoperatively, 31 patients $(67.39 \%)$ did not show any LIV resection-related complications, 15 patients $(32.61 \%)$ showed mild LIV resection-related complications, and no patients $(0.00 \%)$ showed severe LIV resection-related complications.

\section{Factors affecting the safety of LIV resection}

Based on the safety of LIV resection, the patients were divided into 2 groups: a "Safety" group and a "Relative Safety" group. Analyses of the 2 groups indicated statistically significant differences in LIV invasion and its severity $(\mathrm{P}=0.01)$, surgical procedure $(\mathrm{P}<0.01)$, left halfperimeter and right half-perimeter of the anterior chest wall at days 1,3 and 5 after surgery $(\mathrm{P}<0.01)$, and left median cubital venous pressure at days 1,3 and 5 after surgery $(\mathrm{P}<0.01)$ but showed no differences in sex, age, $\mathrm{BMI}$, preoperative platelet count, preoperative hemoglobin, preoperative comorbidities, intraoperative blood loss, operative time, average hospital stay, postoperative pathology, and postoperative complications (Table 1). Multivariate logistic regression analysis suggested that the degree of LIV invasion and surgical approach were risk factors for predicting LIV resection safety.

\section{Comparison of perioperative outcomes of "three-bole" laparoscopic surgery via the subxiphoid and subcostal arch approach and open surgery via a vertical incision in the sternum}

The comparison of perioperative outcomes of "threehole" laparoscopic surgery via the subxiphoid and subcostal arch approach and that of the open surgery via a vertical incision in the sternum indicated no differences in sex, age, BMI, degree of preoperative LIV invasion, preoperative platelet count, preoperative hemoglobin level, preoperative 
Table 1 Univariate analysis of clinical data and safety of LIV resection

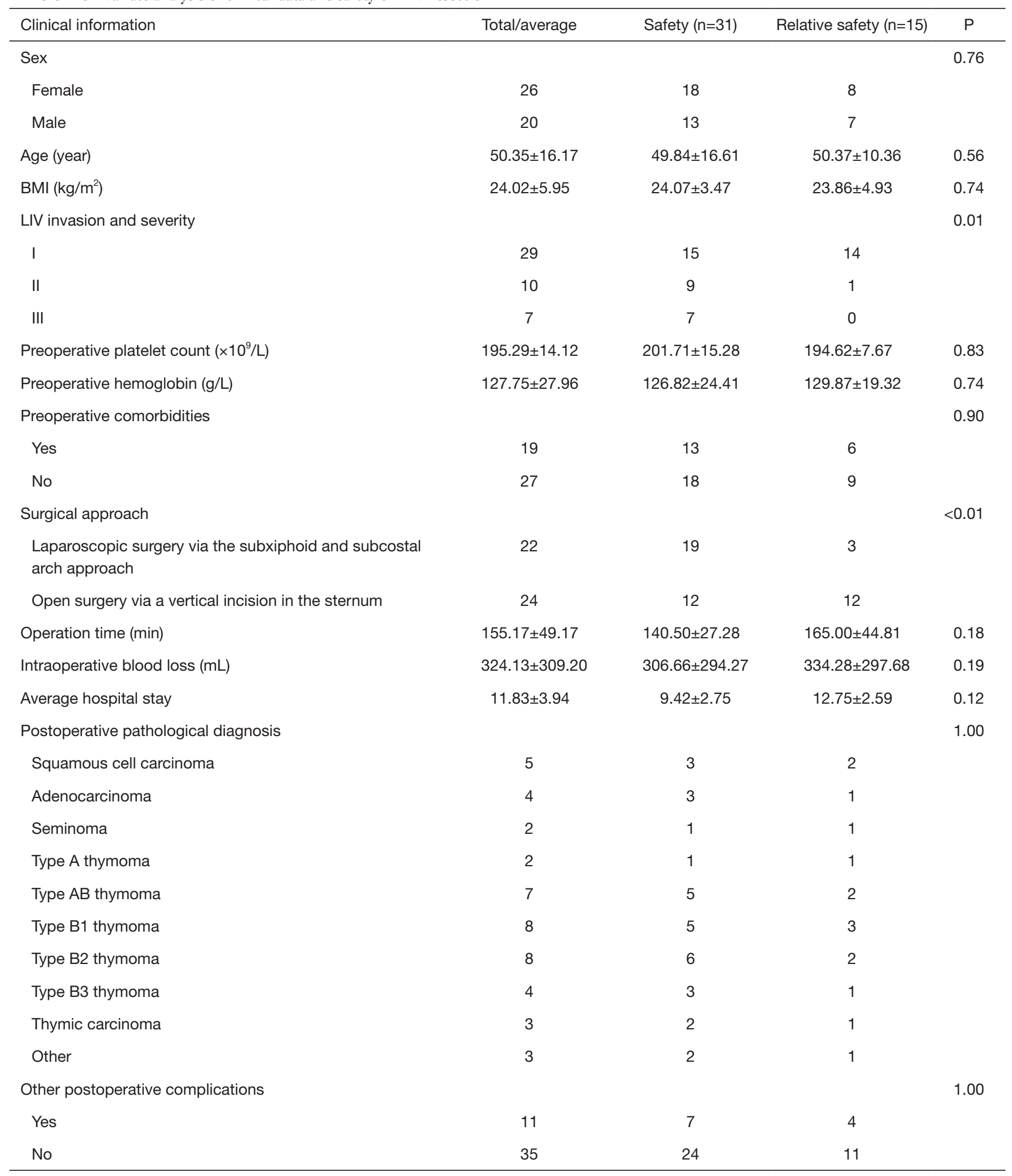

Table 1 (continued) 
Table 1 (continued)

\begin{tabular}{|c|c|c|c|c|}
\hline Clinical information & Total/average & Safety $(n=31)$ & Relative safety $(n=15)$ & $P$ \\
\hline \multicolumn{5}{|c|}{ Difference of the median circumference between the left and the right forearm $(\mathrm{mm})$} \\
\hline 1 day before surgery & $0.11 \pm 0.10$ & $0.05 \pm 0.13$ & $0.03 \pm 0.01$ & 0.36 \\
\hline 1 day after surgery & $2.12 \pm 1.78$ & $0.17 \pm 0.14$ & $8.85 \pm 4.78$ & $<0.01$ \\
\hline 3 days after surgery & $4.18 \pm 2.12$ & $0.18 \pm 0.14$ & $12.98 \pm 6.55$ & $<0.01$ \\
\hline 5 days after surgery & $4.01 \pm 1.65$ & $0.17 \pm 0.17$ & $10.54 \pm 6.38$ & $<0.01$ \\
\hline 30 days after surgery & $0.10 \pm 0.07$ & $0.07 \pm 0.11$ & $0.18 \pm 0.13$ & 0.82 \\
\hline \multicolumn{5}{|c|}{ Left median cubital venous pressure $\left(\mathrm{cmH}_{2} \mathrm{O}\right)$} \\
\hline 1 day before surgery & $10.34 \pm 4.63$ & $12.04 \pm 3.78$ & $8.84 \pm 2.46$ & 0.24 \\
\hline 1 day after surgery & $16.56 \pm 5.98$ & $14.83 \pm 4.45$ & $20.36 \pm 8.19$ & $<0.01$ \\
\hline 3 days after surgery & $22.84 \pm 5.24$ & $18.64 \pm 9.93$ & $27.33 \pm 8.56$ & $<0.01$ \\
\hline 5 days after surgery & $21.04 \pm 7.11$ & $18.01 \pm 8.76$ & $25.65 \pm 6.83$ & $<0.01$ \\
\hline 30 days after surgery & $13.54 \pm 5.24$ & $13.31 \pm 4.48$ & $13.75 \pm 4.27$ & 0.64 \\
\hline Follow-up time/months & $32.34 \pm 13.10$ & $32.21 \pm 13.35$ & $33.25 \pm 13.74$ & 0.65 \\
\hline
\end{tabular}

comorbidities, and postoperative pathology but showed significant differences in intraoperative blood loss $(\mathrm{P}<0.01)$, operation time $(\mathrm{P}<0.01)$, average hospital stay $(\mathrm{P}<0.01)$, and other postoperative complications $(\mathrm{P}<0.01)$ (Table 2).

\section{Discussion}

Anterior mediastinal tumors often involve the superior vena cava and the left and right innominate veins and cause superior vena cava syndrome in severe cases $(3-5,17)$. Artificial vascular replacement or pericardial patch repair are difficult and risky, and in the 1970s, superior vena cava syndrome was often listed as a surgical contraindication, for which conservative treatment was recommended (18). After nearly 4 decades, with the improvement in diagnostic and surgical techniques and the extensive application of vascular substitutes, breakthroughs have been made regarding operations on patients with superior vena cava syndrome, and the removal of the superior vena cava and (or) the left and right innominate veins and vessel replacement or bypass through artificial blood vessels have made it possible for radical resection of tumors or have relieved superior vena cava obstruction $(11,12,17,19-23)$. For cases in which the anterior mediastinal lesion simply invades the LIV and phleboplasty cannot be performed, what are the options? Artificial vascular replacement or LIV resection? The former conforms to the physiology and is conducive to blood return but is associated with unfavorable factors such as surgical trauma, long operation time, high surgical difficulty, and the need for long-term anticoagulation; the latter is simple, fast, and minimally invasive, but its safety and postoperative blood return pathway have not been thoroughly investigated $(6,15,16)$ but were explored in this study.

We found that postoperatively, $67.39 \%$ of patients with LIV resection did not show any LIV resection-related complications and that $32.61 \%$ of the patients showed mild LIV resection-related complications, mostly left upper limb swelling, mild headache, and dizziness; no patient $(0.00 \%)$ showed severe LIV resection-related complications, e.g., tissue necrosis in the left upper limb, sensory and motor dysfunction disorders, convulsions, impaired vision and disturbance of consciousness, etc. The symptoms were more severe on days 3, 4 and 5 after surgery and then gradually diminished, lasting approximately 1 month, which was consistent with the changes in the Difference of the median circumference between the left and the right forearm and the left median cubital venous pressure at days 1, 3, 5 and 30 after surgery.

The LIV is formed by the confluence of the left internal jugular vein and left subclavian vein posterior of the left sternoclavicular joint, accommodating venous blood flow in the left internal jugular vein, left subclavian vein, left vertebral vein, left internal thoracic vein, and inferior 
Table 2 Comparison of the perioperative outcomes of the "three-hole" laparoscopic surgery via the subxiphoid and subcostal arch approach and those of open surgery via a vertical incision in the sternum

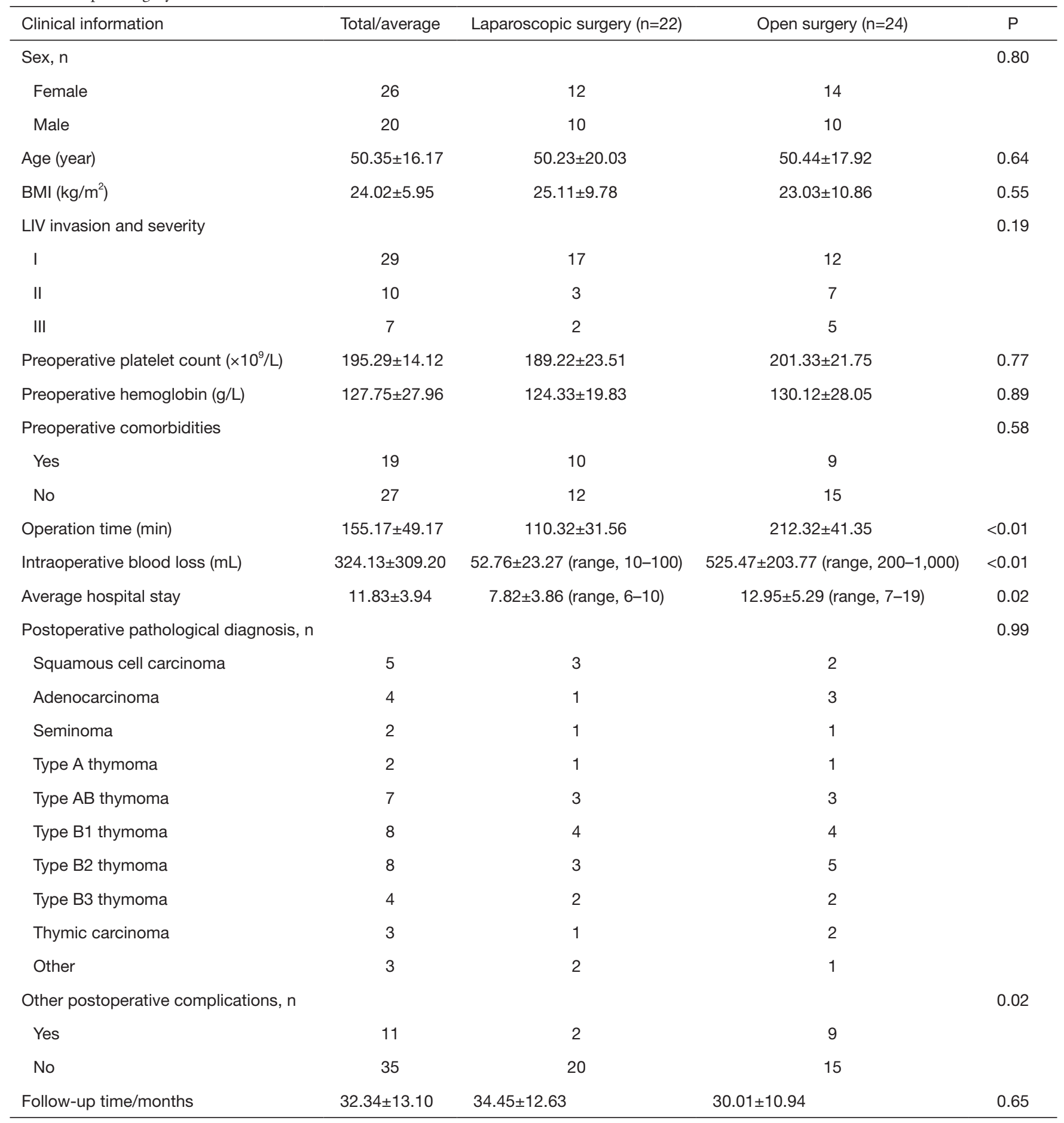

thyroid vein and collecting venous blood flow from the left head and neck, left upper limb and left chest (except the heart) and part of the left upper abdominal wall (15). After
LIV resection, the related blood return pathways may be as follows: (I) communicating branches of the left and right innominate vein: the left and right innominate veins have 
Table 3 Analysis of the relationship between the degree of LIV stenosis and the difference of the median circumference between the left and the right forearm and that between the degree of LIV stenosis and the left median cubital venous pressure

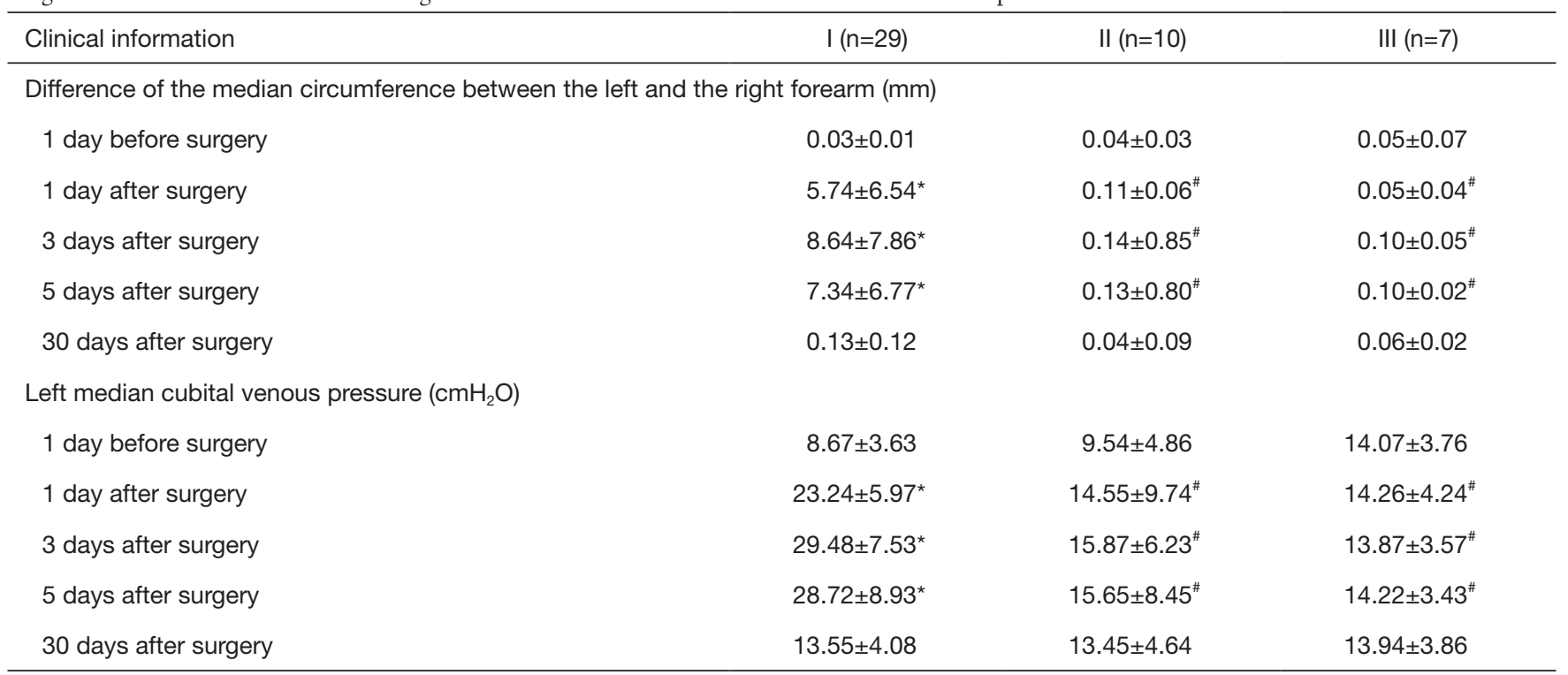

\#, $\mathrm{P}<0.05$ vs. I ( $\mathrm{n}=29) ;{ }^{*}, \mathrm{P}<0.05$ vs. 1 day before surgery.

extensive communicating branches in the cranium, the face, the neck, and between the deep and superficial veins of the upper chest wall, and when the LIV is chronically obstructed, the venous blood collected by the LIV from the left head and neck, the left chest, and part of the left upper abdominal wall can be returned to the right innominate vein (24); (II) communicating branches of the superior and inferior vena cava (Figure 3): there are several important communicating branches between the superior and inferior vena cava (16,25-27), including the azygos venous system, the internal thoracic and pericardiacophrenic veins, and the lateral thoracic and thoracic wall and abdominal wall veins $(28,29)$, of which the azygos venous system consists of the azygos vein, accessory azygos vein, and accessory hemiazygos vein and mainly collects blood flow from the shoulder and back veins, the vertebral venous plexus and the posterior intercostal veins and connects the LIV and the superior and inferior vena cava. The communicating branches of the superior vena cava and the azygous venous system are important pathways for acute LIV obstruction and venous return in the left upper limb (Figures 3-5).

In this study, we found that the preoperative degree of LIV stenosis is an important factor for the safety of LIV resection. Based on the results in Table 3, with grades II and III LIV stenosis, the Difference of the median circumference between the left and the right forearm and the left median cubital venous pressure on days 1,3 , or
5 were not significantly different from those the day before surgery, suggesting that in the case of severe or complete obstruction of LIV, blood return and venous communicating branches have already formed preoperatively; therefore, LIV removal has no effect on blood circulation. In the case of grade I LIV stenosis, the Difference of the median circumference between the left and the right forearm and the left median cubital venous pressure on days 1,3 , or 5 were significantly greater than those the day before surgery. Furthermore, for grades II and III LIV stenosis, the results suggest that with moderate LIV stenosis, the removal of the LIV may cause LIV resection-related complications in the formation process of collateral circulation after surgery.

With the rapid development of thoracoscopic techniques, video-assisted thoracoscopic surgery (VATS) has been widely used in the resection of anterior mediastinal lesions. Compared with traditional open surgery, thoracoscopic surgery has the advantages of less trauma, less bleeding, quicker recovery, fewer complications, etc. and is thus a safer and more effective procedure. In this study, we also found that compared with open surgery via a vertical incision in the sternum, laparoscopic surgery had a shorter operation time, less intraoperative blood loss, and shorter average hospital stay, with fewer LIV resection-associated complications (Table 2). In particular, "three-hole" laparoscopic surgery via the subxiphoid and subcostal arch approach (30-34) can reduce damage to the communicating 


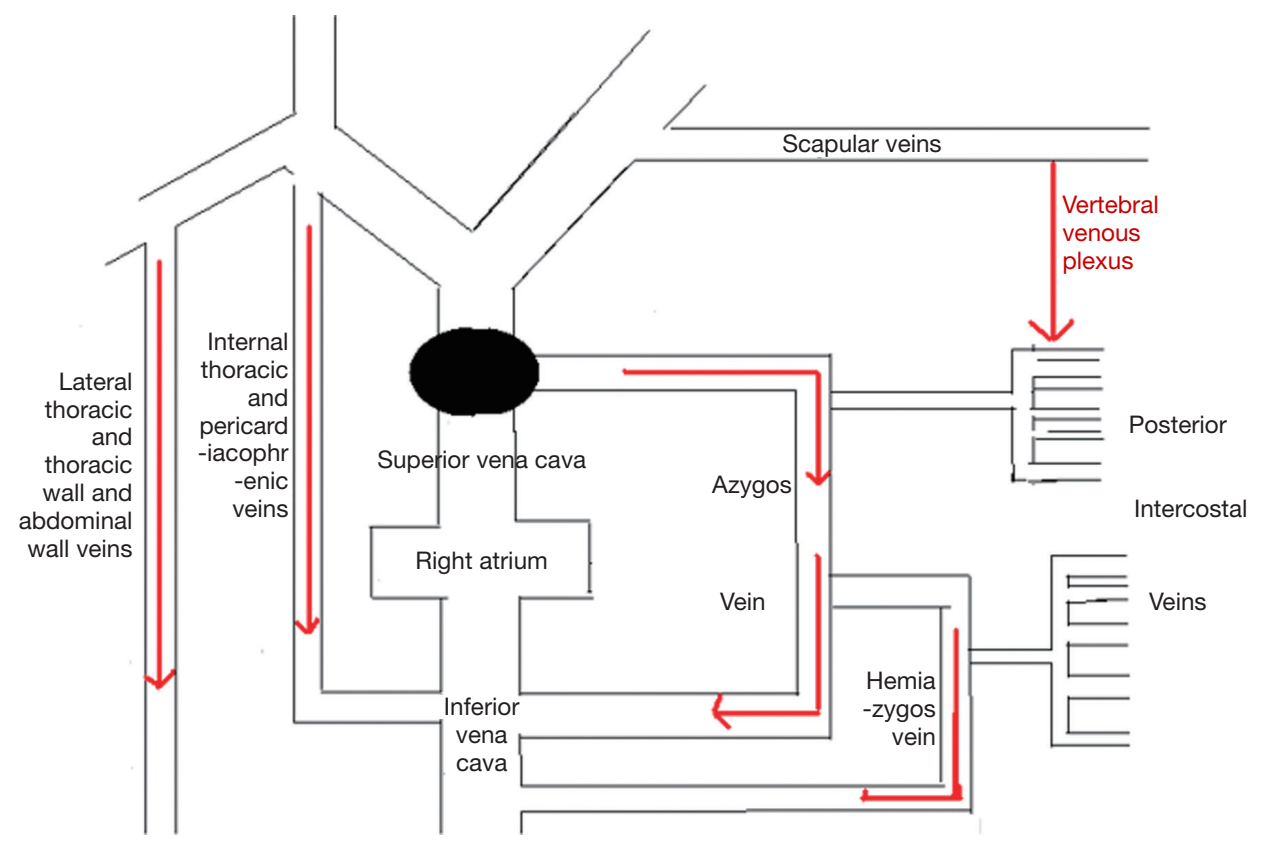

Figure 3 Communicating branches of the superior and inferior vena cava.

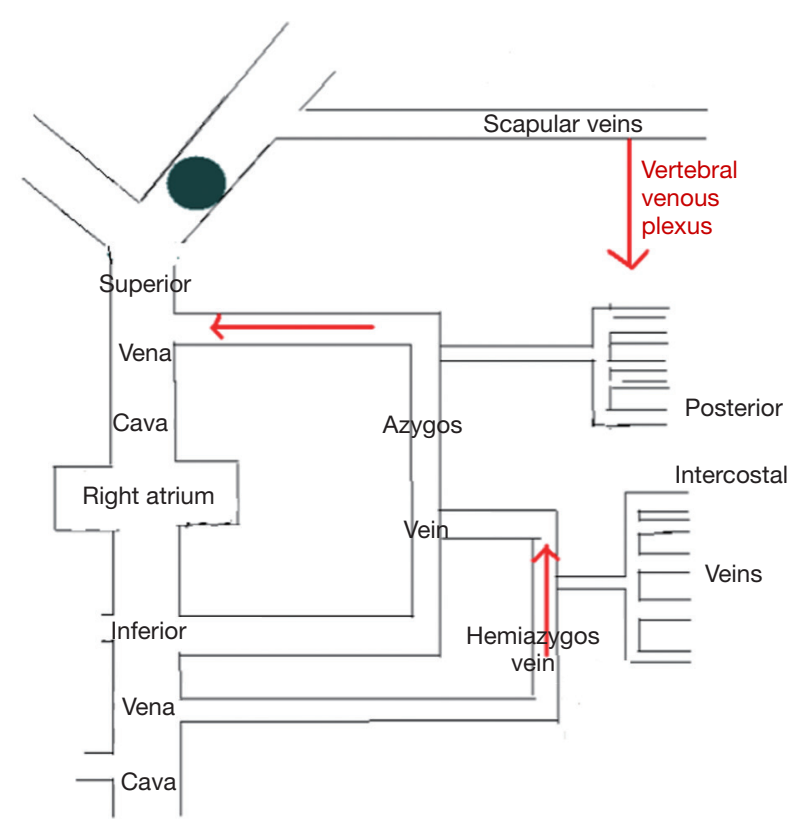

Figure 4 Azygos venous system.

branches of the chest wall and is conducive to the protection of the left internal thoracic vein and the pericardiacophrenic vein under direct vision during surgery, which reduces the incidence of postoperative blockage of blood return. Certainly, the choice of surgical approach is more dependent on the location and size of lesions in the anterior mediastinum, and under the premise of full assessment of the preoperative conditions and when conditions permit, laparoscopic surgery via the subxiphoid and subcostal arch approach should be clinically promoted.

\section{Conclusions}

Our extensive literature survey indicated that for superior vena cava obstruction, artificial vascular replacement has been mostly adopted while simple LIV resection has been mentioned rarely, without examination of its safety $(6,15,16)$. In this study, we retrospectively analyzed the clinical cases of anterior mediastinal lesion and LIV resection in our hospital and concluded that LIV resection is a relatively simple, safe and effective surgical procedure. Although some patients may develop mild complications such as left upper limb swelling and dizziness, the complications can be effectively treated with diuretics, reduction in intracranial pressure and anticoagulation therapy. The degree of LIV invasion and laparoscopic surgery are safe factors for LIV resection-associated complications. Because this study is a retrospective analysis, there are limitations such as bias, and the findings should be verified through multicenter, largescale prospective randomized controlled trials. 


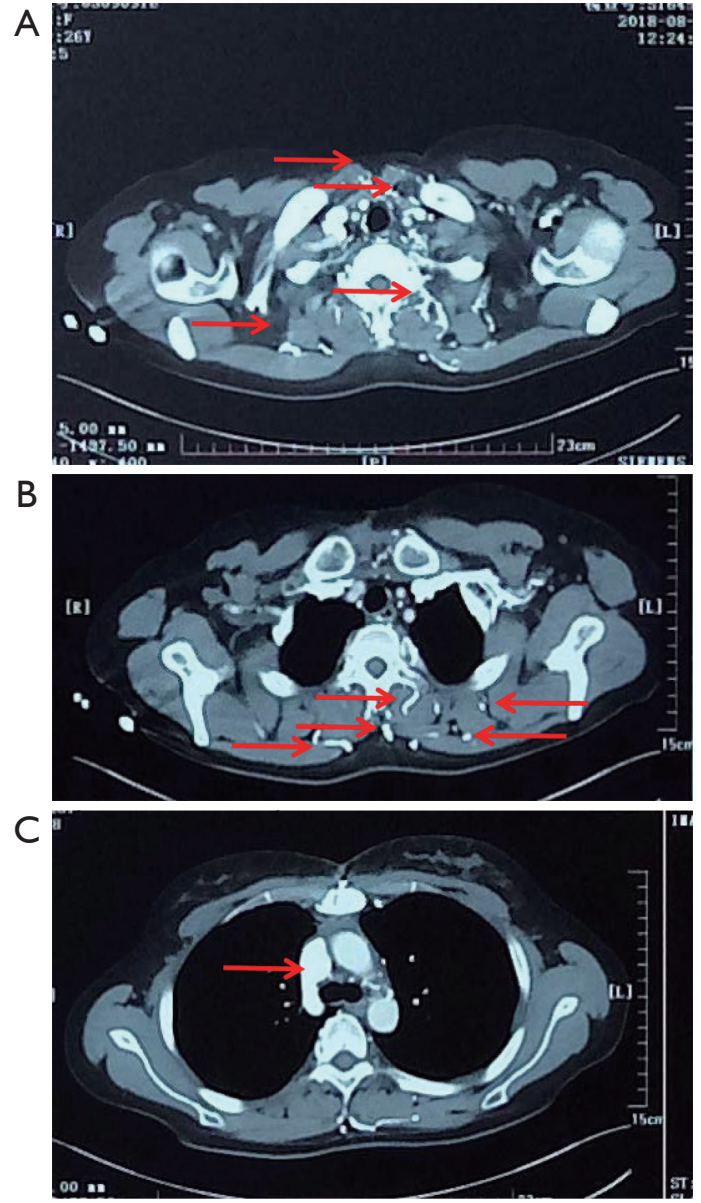

Figure 5 Images from a 27-year-old woman taken at the time of reexamination 1 month after anterior mediastinal lesion and LIV resection. Her postoperative pathological diagnosis was type B2 thymoma. (A) Communicating branches of the deep and superficial veins of the neck; (B) communicating branches of veins of the shoulder and back; (C) thickened azygos vein.

\section{Acknowledgments}

Funding: This study was partially supported by Key Research and Development Plan of Shaanxi Province (No. 2017ZDXM-SF-052).

\section{Footnote}

Conflicts of Interest: The authors have no conflicts of interest to declare.

Ethical Statement: The authors are accountable for all aspects of the work in ensuring that questions related to the accuracy or integrity of any part of the work are appropriately investigated and resolved. Our Ethical Committee was informed of the study and did not require approval. All patients gave their informed consent for the study.

Open Access Statement: This is an Open Access article distributed in accordance with the Creative Commons Attribution-NonCommercial-NoDerivs 4.0 International License (CC BY-NC-ND 4.0), which permits the noncommercial replication and distribution of the article with the strict proviso that no changes or edits are made and the original work is properly cited (including links to both the formal publication through the relevant DOI and the license). See: https://creativecommons.org/licenses/by-nc-nd/4.0/.

\section{References}

1. Engels EA, Pfeiffer RM. Malignant thymoma in the United States: demographic patterns in incidence and associations with subsequent malignancies. Int J Cancer 2003;105:546-51.

2. Giaccone G. Treatment of malignant thymoma. Curr Opin Oncol 2005;17:140-6.

3. Funakoshi $\mathrm{Y}$, Ohta M, Maeda H, et al. Extended operation for invasive thymoma with intracaval and intracardiac extension. Eur J Cardiothorac Surg 2003;24:331-3.

4. Davenport E, Malthaner RA. The role of surgery in the management of thymoma: a systematic review. Ann Thorac Surg 2008;86:673-84.

5. Dong S, Lv G, Chen L, et al. Report on a case of malignant thymoma extending to the right atrium through the innominate vein. Chin J Thorac Cardiov Surg 2004;20:62.

6. Hoshino $\mathrm{H}$, Matsunaga T, Takamochi $\mathrm{K}$, et al. Is postoperative anticoagulation necessary after left innominate vein division in general thoracic surgery? Gen Thorac Cardiovasc Surg 2019;67:254-8.

7. Spaggiari L, Magdeleinat P, Kondo H, et al. Results of superior vena cava resection for lung cancer. Analysis of prognostic factors. Lung Cancer 2004;44:339-46.

8. Grunenwald DH. Resection of lung carcinomas invading the mediastinum, including the superior vena cava. Thorac Surg Clin 2004;14:255-63.

9. Bretti S, Berruti A, Loddo C, et al. Multimodal management of stages III-IVa malignant thymoma. Lung Cancer 2004;44:69-77. 
10. Kondo K, Monden Y. Therapy for thymic epithelial tumors: a clinical study of 1;320 patients from Japan. Ann Thorac Surg 2003;76:878-84; discussion 884-5.

11. Leo F, Bellini R, Conti B, et al. Superior vena cava resection in thoracic malignancies: does prosthetic replacement pose a higher risk?. Eur J Cardiothorac Surg 2010;37:764-9.

12. Arvind K, Roman D, Umashankkar K, et al. Resection and reconstruction of mediastinal great vessels in invasive thymoma. Indian J Cancer 2010;47:400-405.

13. Borri A, Leo F, Veronesi G, et al. Extended pneumonectomy for non-small cell lung cancer: morbidity, mortality, and long-term results. J Thorac Cardiovasc Surg 2007;134:1266-72.

14. Misthos P, Papagiannakis G, Kokotsakis J, et al. Surgical management of lung cancer invading the aorta or the superior vena cava. Lung Cancer 2007;56:223-7.

15. Sai Sudhakar CB, Elefteriades JA. Safety of left innominate vein division during aortic arch surgery. Ann Thorac Surg 2000;70:856-8.

16. McPhee A, Shaikhrezai K, Berg G. Is it safe to divide and ligate the left innominate vein in complex cardiothoracic surgeries?. Interact Cardiovasc Thorac Surg 2013;17:560-3.

17. Gebhardt C, Kohler J, Gentsch HH, et al. Resection of the superior vena cava in tumor occlusion. Chirurg 1989;60:529-35.

18. Hsu JW, Chiang CD, Hsu WH, et al. Superior vena cava syndrome in lung cancer: an analysis of 54 cases. Gaoxiong Yi Xue Ke Xue Za Zhi 1995;11:568-73.

19. Spaggiari L, Thomas P, Magdeleinat $P$, et al. Superior vena cava resection with prosthetic replacement for non-small cell lung cancer: long-term results of a multicentric study. Eur J Cardiothorac Surg 2002;21:1080-6.

20. Li F, Zhao F, Guo Y, et al. Treatment of tumors invading the superior vena cava and branches. Chin J Thorac Cardiov Surg 2000;16:44-5.

21. Zhou Q. Indication and advances of extended resection of locally advanced lung cancer. Chin Oncol 2003;25:22-6.

22. Zhou Q, Liu L, Liu B, et al. Lobectomy or pneumonectomy combined with extended resection of the heart, great vessels in the treatment of locally advanced lung cancer. Chin J Lung Cancer 2001;4:403-6.

23. Wang Z, Yu H, Ren H, et al. Extended Resection and Reconstruction of Superior Vena Cava and Innominate Vein for Mediastinal Tumor. Chin J Clin Thorac Cardiov Surg 2005;12:22-5.

24. Yonei A, Sari A. Reversal of blood flow in the internal jugular vein. Anesthesiology 1991;74:634-5.

25. Bashist B, Parisi A, Frager DH, et al. Abdominal CT findings when the superior vena cava, brachiocephalic vein, or subclavian vein is obstructed. AJR Am J Roentgenol 1996;167:1457-63.

26. Liu C, Miao J, He Z, et al. Spiral CT study of superior vena cava syndrome: correlation between collateral pathways and obstruction site and degree. Zhongguo Fei Ai Za Zhi 2001;4:347-50.

27. Simeone FJ, Bennett DL, Chang CY, et al. Retrospective analysis of intravertebral collateral enhancement in patients with central venous obstruction. Skeletal Radiol 2016;45:163-8.

28. Kim HC, Chung JW, Yoon CJ, et al. Collateral pathways in thoracic central venous obstruction: three-dimensional display using direct spiral computed tomography venography. J Comput Assist Tomogr 2004;28:24-33.

29. Politi L, Crisci C, Montinaro F, et al. Prosthetic replacement and tangential resection of the superior vena cava in chest tumors. J Cardiovasc Surg (Torino) 2007;48:363-8.

30. Lu Q, Li X, Zhou Y, et al. Thymus resection with " three holes" operation through subxiphoid and subcostal in the treatment of myasthenia gravis. Chin J Thorac Surg 2016;3:95-9.

31. Zhao Z, Wang H, Zhou Y, et al. Recent Progress of Minimally Invasive Surgery in Anterior Mediastinal Diseases. Medical Recapitulate 2016;22:4856-9.

32. Zhao Z, Yin X, Zhou Y. Clinical application of harmonic scalpel in subxiphoid and subcostal arch approach for resection of anterior mediastinal lesion. Chin J Clin Thorac Cardiov Surg 2018;25:23-7.

33. Zhao J, Wang J, Zhou Y, et al. Subxiphoid and subcostal arch thoracoscopic extended thymectomy: a safe and feasible minimally invasive procedure for selective stage III thymomas. J Thorac Dis 2016;8:S258-64.

34. Lu Q, Zhao J, Zhou Y, et al. Subxiphoid and subcostal arch "Three ports" thoracoscopic extended thymectomy for myasthenia gravis. J Thorac Dis 2018;10:1711-20.

Cite this article as: Wang HQ, Tian F, Wei M, Chen LH, Cheng SY, Feng Z, Ma J, Zhang TY, Guo YZ, Yin XL, Zhao ZW, Zhou YA. Preliminary evaluation and discussion of the safety of left innominate vein resection. J Thorac Dis 2020;12(3):438-447. doi: 10.21037/jtd.2020.01.29 